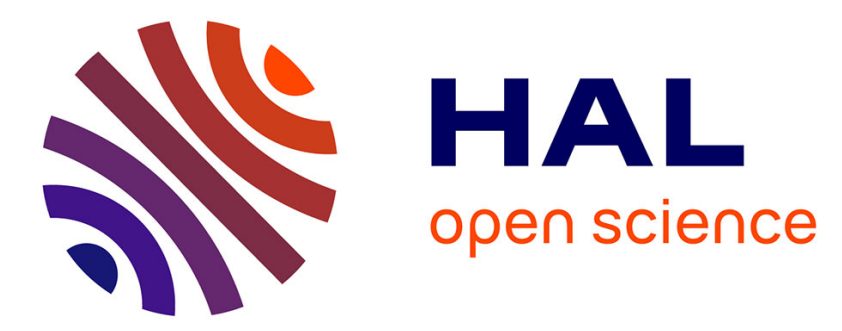

\title{
The implicit structure of ridges of a smooth parametric surface
}

Frédéric Cazals, Jean-Charles Faugère, Marc Pouget, Fabrice Rouillier

\section{To cite this version:}

Frédéric Cazals, Jean-Charles Faugère, Marc Pouget, Fabrice Rouillier. The implicit structure of ridges of a smooth parametric surface. Computer Aided Geometric Design, 2006, 23 (7), pp.582-598. 10.1016/j.cagd.2006.04.002 . inria-00097581

\section{HAL Id: inria-00097581 https://hal.inria.fr/inria-00097581}

Submitted on 21 Sep 2006

HAL is a multi-disciplinary open access archive for the deposit and dissemination of scientific research documents, whether they are published or not. The documents may come from teaching and research institutions in France or abroad, or from public or private research centers.
L'archive ouverte pluridisciplinaire HAL, est destinée au dépôt et à la diffusion de documents scientifiques de niveau recherche, publiés ou non, émanant des établissements d'enseignement et de recherche français ou étrangers, des laboratoires publics ou privés. 


\title{
The implicit structure of ridges of a smooth parametric surface
}

\author{
Frédéric Cazals Jean-Charles Faugère Marc Pouget Fabrice Rouillier *
}

May 2, 2006

\begin{abstract}
Given a smooth surface, a blue (red) ridge is a curve such that at each of its points, the maximum (minimum) principal curvature has an extremum along its curvature line. Ridges are curves of extremal curvature and therefore encode important informations used in segmentation, registration, matching and surface analysis. State of the art methods for ridge extraction either report red and blue ridges simultaneously or separately —in which case a local orientation procedure of principal directions is needed, but no method developed so far certifies the topology of the curves reported.

On the way to developing certified algorithms independent from local orientation procedures, we make the following fundamental contributions. For any smooth parametric surface, we exhibit the implicit equation $P=0$ of the singular curve $\mathscr{P}$ encoding all ridges and umbilics of the surface (blue and red), and show how to recover the colors from factors of $P$. Exploiting second order derivatives of the principal curvatures, we also derive a zero dimensional system coding the so-called turning points, from which elliptic and hyperbolic ridge sections of the two colors can be derived. Both contributions exploit properties of the Weingarten map of the surface in the specific parametric setting and require computer algebra. Algorithms exploiting the structure of $P$ for algebraic surfaces are developed in a companion paper.
\end{abstract}

\section{Introduction}

\subsection{Ridges}

Differential properties of smooth surfaces embedded in $\mathbb{R}^{3}$ are a fascinating topic per se, and have long been of interest for artists and mathematicians, as illustrated by the parabolic lines drawn by Felix Klein on the Apollo of Belvedere [HCV52], and also by the developments reported in [Koe90]. Beyond these noble considerations, the recent development of laser range scanners and medical images shed light on the importance of being able to analyze discrete datasets consisting of point clouds in $3 D$ or medical images — grids of $3 D$ voxels. Whenever the datasets processed model piecewise smooth surfaces, a precise description of the models naturally calls for differential properties. In particular, applications such as shape matching [ $\mathrm{HGY}^{+}$99], surface analysis [HGY ${ }^{+}$99], or registration [PAT00] require the characterization of high order properties and in particular the characterization of curves of extremal curvatures, which are precisely the so-called ridges. Interestingly, ridges are also ubiquitous in the analysis of Delaunay based surface meshing algorithms [ABL03].

A comprehensive description of ridges can be found in [Por71, $\mathrm{HGY}^{+} 99$, Por01, CP05a], and in the sequel, we just introduce the basic notions so as to discuss our contributions. Consider a smooth embedded surface, and denote $k_{1}$ and $k_{2}$ the principal curvatures, with $k_{1} \geq k_{2}$. Umbilics are the points where $k_{1}=k_{2}$. For any non umbilical point, the corresponding principal directions of curvature are well defined, and we denote them $d_{1}$ and $d_{2}$. In local coordinates, we denote $\langle$,$\rangle the inner product induced by the ambient Euclidean space, and d k_{1}, d k_{2}$ the gradients of the principal curvatures. Ridges are defined by:

Definition. 1 A non umbilical point is called

- a blue ridge point if the extremality coefficient $b_{0}=\left\langle d k_{1}, d_{1}\right\rangle$ vanishes, i.e. $b_{0}=0$.

- a red ridge point if the extremality coefficient $b_{3}=\left\langle d k_{2}, d_{2}\right\rangle$ vanishes, i.e. $b_{3}=0$.

*INRIA. firstname.lastname@inria.fr 
Notations $b_{0}$ and $b_{3}$ refer to the third order coefficients of the taylor expansion of the surface when it is parametrized by the principal directions $\left[\mathrm{HGY}^{+} 99, \mathrm{CP} 05 \mathrm{a}\right]$.

Notice that, as the principal curvatures are not differentiable at umbilics, the extremality coefficients are not defined at such points. In addition, the sign of the extremality coefficients is not defined since the principal directions can be oriented by two opposite unit vectors. Apart from umbilics, special points on ridges are purple points, which correspond to intersections between red and a blue ridges. The previous characterization of ridges involves third-order differential properties. Using fourth-order differential quantities, a ridge point can further be qualified as elliptic if it corresponds to a maximum of $k_{1}$ or a minimum of $k_{2}$, or hyperbolic otherwise. Ridges of a given color change from elliptic to hyperbolic at special points called turning points.

The calculation of ridges poses difficult problems, which are of three kinds.

Topological difficulties. Ridges of a smooth surface form a singular curve on the surface, with self-intersections at umbilics (more precisely at so-called 3-ridges umbilics), and purple points. Moreover, ridges have complex interactions with curvature lines at turning points. From the application standpoint, reporting ridges of a surface faithfully requires reporting umbilics, purple points and turning points.

Numerical difficulties. As pointed out above, ridges are characterized and qualified through third and fourth order derivatives of the surface. Estimating them depends on the particular type of surface processed -implicitly defined, parameterized, discretized by a mesh — and is numerically a difficult task.

Orientation difficulties. Since coefficients $b_{0}$ and $b_{3}$ depend on a given orientation of the principal directions, their sign is not well defined. Practically, tracking the sign change of functions whose sign depends on the particular orientation of the frame in which they are expressed poses a problem. In particular, tracking a zero-crossing of $b_{0}$ or $b_{3}$ from sign changes along a curve segment on the surface imposes to find a coherent orientation of the principal frame at the endpoints. Given two principal directions at these endpoints, one way to find a local orientation consists of choosing two vectors so that they make an acute angle, whence the name Acute Rule. This rule has been used since the very beginning of computer examination of ridges [Mor90, Mor96], and is implicitly used in almost all algorithms. But the question of specifying conditions guaranteeing the decisions made are correct has only been addressed recently [CP05b].

An other approach is to extract the zero level set of the Gaussian extremality $E_{g}=b_{0} b_{3}$ defined in [Thi96]. This function has a well defined sign independent from the orientation, but it is still not defined at umbilics and one cannot distinguish blue from red ridges.

\subsection{Paper overview}

The paper is organized as follows. The positioning of our paper with respect to the traditional characterization of ridges in singularity theory is discussed in section 2 . Notations are set in section 3 , and preliminary differential lemmas are proved in section 4 . The implicit equation for ridges and umbilics is derived in section 5 . The system for turning points and the determination of ridge types are stated in section 6 . Corollaries for polynomial parametric surfaces and an illustration of the effectiveness of the main theorem on a complex Bezier surface is given in section 7. Some of the proofs require symbolic computations, which we perform with Maple, and provide in appendix 9 -also available on the web page of the authors [CFPR].

\section{Ridges: the local and global viewpoints}

\subsection{Global characterization of ridges using contact theory}

Ridges can be characterized either as extrema of principal curvatures along their curvature lines as in definition 1 , or by analyzing the contact between the surface and spheres [ $\left.\mathrm{HGY}^{+} 99\right]$. For parametric surfaces, this later approach allows a global characterization of ridges [Por01, Chapter 11], and we therefore state the main result so as to position our contribution. 
Consider a parameterized surface $S$, with parameterization $s: D \subset \mathbb{R}^{2} \rightarrow \mathbb{R}^{3}$. For any point $c \in \mathbb{R}^{3}$, the contact function between the surface and the sphere centered at $c$ is defined by:

$$
V(c)=\langle c, s\rangle-\frac{1}{2}\langle s, s\rangle .
$$

To see the connection with definition 1 , let $V(c)_{k}$ denote the $k$-times linear form of the derivative of order $k$ w.r.t. $w$ associated to point $c$. Ridges are essentially characterized in the following theorem [Por01, Thm 11.10 page 191]:

Theorem. 1 Let s be a regular surface in $R^{3}$ with only ordinary umbilics. Then these points $(c, w, u)$ of $\mathbb{R}^{3} \times \mathbb{R}^{2} \times$ $\mathbb{R}^{2}$ where

$$
V(c)_{1}(w)=0, V(c)_{2}(w) u=0, V(c)_{3}(w) u^{3}=0,\|u\|=1
$$

form a smooth submanifold of $\mathbb{R}^{3} \times \mathbb{R}^{2} \times \mathbb{R}^{2}$ of dimension 1 , with certain exceptions that will appear in the course of the proof.

For a point $(c, w, u)$ on this submanifold, $c$ is a rib point (singularity of the focal surfaces) and $s(w)$ is a ridge point. If $s(w)$ is not an umbilic, $u$ is a vector whose direction is a principal direction. Notice that to characterize a ridge point, one needs to find (i) a point $c$ in the ambient space of the surface, (ii) a point $w$ in the parameterization domain, (iii) a vector $u$ in the parameterization domain.

\subsection{Global characterization of ridges using extremality coefficients}

Using contact theory, theorem 1 states that generically, ridges are described by a smooth curve in a seven dimensional space. Instead, using the characterization of ridges through extremality coefficients, we show that ridges can be described by a singular curve in the two-dimensional parametric domain of the surface. The relationship between these two descriptions is easily understood: by working in the seven dimensional space, Porteous avoids the self-intersections of the ridge curve - these intersections appear on the surface or in the parametric domain. Two additional comments are in order. First, implicitizing this codimension six one-dimensional manifold is a priori not trivial, and has not been done to the best of our knowledge. Second, instead of working with the squared distance function, we use the alternative description of ridges in terms of extrema of curvatures, which has the advantage of eluding the dependence to the center of the sphere with which the contact to the surface is studied.

More precisely, let $\Phi(u, v)$ be a smooth parameterized surface over a domain $\mathscr{D} \subset \mathbb{R}^{2}$. We exhibit the implicit equation $P=0$ of the singular curve $\mathscr{P}$ encoding all ridges of the surface (blue and red) and umbilics, and show how to recover the colors from factors of $P$. We also derive a zero dimensional system coding the so-called turning points, from which elliptic and hyperbolic ridge sections of the two colors can be derived.

To conclude, it should be emphasized that our contribution is fundamentally different from previous work. With respect to [Por01], we have a global implicit formulation as a curve in a $2 D$ domain. With respect to [ $\mathrm{HGY}^{+} 99$ ] -in particular the analysis carried out using extremality coefficients, we provide a global rather than local analysis of the ridge curve. With respect to [Mor90, Mor96], we do not resort to local orientation procedures. In particular, our contributions lead to the design of the first certified algorithm for reporting ridges on polynomial parametric surfaces [CFPR05].

\section{Notations}

Ridges and umbilics. At any non umbilical point of the surface, the maximal (minimal) principal curvature is denoted $k_{1}\left(k_{2}\right)$, and its associated direction $d_{1}\left(d_{2}\right)$. Anything related to the maximal (minimal) curvature is qualified blue (red), for example we shall speak of the blue curvature for $k_{1}$ or the red direction for $d_{2}$. Since we shall make precise statements about ridges, it should be recalled that, according to definition 1, umbilics are not ridge points.

Differential calculus. Let $f(u, v): \mathscr{D} \subset \mathbb{R}^{2} \longrightarrow \mathbb{R}$ be a continuously differentiable function. The derivative of $f$ wrt variable $u$ denoted $f_{u}$, we identify linear forms and gradient vectors so that for $x \in T_{p} \mathbb{R}^{2}, d f_{p}(x)=\left\langle d f_{p}, x\right\rangle$ with $d f_{p}=\left(f_{u}, f_{v}\right)$ in the canonical basis. A point in $\mathscr{D}$ is singular if the gradient $d f$ vanishes, else it is regular. 
Misc. The inner product of two vectors $x, y$ is denoted $\langle x, y\rangle$, the norm of $x$ is $\|x\|=\langle x, x\rangle^{1 / 2}$ and the exterior product is $x \wedge y$.

\section{Manipulations involving the Weingarten map of the surface}

Let $\Phi$ be the parameterization of class $C^{k}$ for $k \geq 4$. Principal directions and curvatures of the surface are expressed in terms of second order derivatives of $\Phi$. More precisely, the matrices of the first and second fundamental forms in the basis $\left(\Phi_{u}, \Phi_{v}\right)$ of the tangent space are

$$
\begin{gathered}
I=\left(\begin{array}{cc}
e & f \\
f & g
\end{array}\right)=\left(\begin{array}{cc}
\left\langle\Phi_{u}, \Phi_{u}\right\rangle & \left\langle\Phi_{u}, \Phi_{v}\right\rangle \\
\left\langle\Phi_{u}, \Phi_{v}\right\rangle & \left\langle\Phi_{v}, \Phi_{v}\right\rangle
\end{array}\right) \\
I I=\left(\begin{array}{cc}
l & m \\
m & n
\end{array}\right)=\left(\begin{array}{cc}
\left\langle N_{n}, \Phi_{u u}\right\rangle & \left\langle N_{n}, \Phi_{u v}\right\rangle \\
\left\langle N_{n}, \Phi_{u v}\right\rangle & \left\langle N_{n}, \Phi_{v v}\right\rangle
\end{array}\right), \quad \text { with } \quad N=\Phi_{u} \wedge \Phi_{v}, \quad N_{n}=\frac{N}{\|N\|} .
\end{gathered}
$$

To compute the principal directions and curvatures, one resorts to the Weingarten map, whose matrix in the basis $\left(\Phi_{u}, \Phi_{v}\right)$ is given by $W=\left(w_{i j}\right)=I^{-1} I I$. The Weingarten map is a self-adjoint operator ${ }^{1}$ of the tangent space [dC76]. The principal directions $d_{i}$ and principal curvatures $k_{i}$ are the eigenvectors and eigenvalues of the matrix $W$. Observing that $\|N\|^{2}=\operatorname{det} I$, matrix $W$ can also be written as follows:

$$
W=\frac{1}{(\operatorname{det} I)^{3 / 2}}\left(\begin{array}{ll}
A & B \\
C & D
\end{array}\right)
$$

This equation defines coefficients $A, B, C$ and $D$ (expressions of special interest for polynomial surfaces, see section 7), for example :

$$
\begin{aligned}
A & =(\operatorname{det} I)^{3 / 2} w_{11}=(\operatorname{det} I)^{3 / 2} \frac{g l-f m}{\operatorname{det} I}=\sqrt{\operatorname{det} I}\left(g\left\langle N / \sqrt{\operatorname{det} I}, \Phi_{u u}\right\rangle-f\left\langle N / \sqrt{\operatorname{det} I}, \Phi_{u v}\right\rangle\right) \\
& =g\left\langle N, \Phi_{u u}\right\rangle-f\left\langle N, \Phi_{u v}\right\rangle .
\end{aligned}
$$

Recall that a parameterized surface is called regular if the tangent map of the parameterization (the Jacobian) has rank two everywhere. Since the first fundamental form is the restriction of the inner product of the ambient space to the tangent space, one has:

Observation. 1 If $\Phi$ is a parameterized surface which is regular, the quadratic form I is positive definite.

In the following, the surface is assumed regular, thus $\operatorname{det}(I) \neq 0$.

\subsection{Principal curvatures.}

The characteristic polynomial of $W$ is

$$
P_{W}(k)=k^{2}-\operatorname{tr}(W) k+\operatorname{det}(W)=k^{2}-\left(w_{11}+w_{22}\right) k+w_{11} w_{22}-w_{12} w_{21} .
$$

Its discriminant is

$$
\Delta(k)=(\operatorname{tr}(W))^{2}-4 \operatorname{det}(W)=\left(w_{11}+w_{22}\right)^{2}-4\left(w_{11} w_{22}-w_{12} w_{21}\right)=\left(w_{11}-w_{22}\right)^{2}+4 w_{12} w_{21} .
$$

A simplification of this discriminant leads to the definition of the following function, denoted $p_{2}$ :

$$
p_{2}=(\operatorname{det} I)^{3} \Delta(k)=(A-D)^{2}+4 B C
$$

The principal curvatures $k_{i}$, with the convention $k_{1} \geq k_{2}$, are the eigenvalues of $W$, that is:

$$
k_{1}=\frac{A+D+\sqrt{p_{2}}}{2(\operatorname{det} I)^{3 / 2}} \quad k_{2}=\frac{A+D-\sqrt{p_{2}}}{2(\operatorname{det} I)^{3 / 2}} .
$$

A point is called an umbilic if the principal curvatures are equal. One has:

\footnotetext{
${ }^{1}$ A self-adjoint map $L$ over a vector space $V$ with a bilinear form $\langle.,$.$\rangle is a linear map such that \langle L u, v\rangle=\langle u, L v\rangle$, for all $u, v \in V$. Such a map can be diagonalized in an orthonormal basis of $V$.
} 
Lemma. 1 The two following equivalent conditions characterize umbilics:

1. $p_{2}=0$

2. $A=D$ and $B=C=0$.

Proof. Since $\operatorname{det}(I) \neq 0$, one has $p_{2}=0 \Leftrightarrow \Delta(k)=0$, hence 1 . characterizes umbilics. Condition 2. trivially implies 1 . To prove the converse, assume that $p_{2}=0$ i.e. the Weingarten map has a single eigenvalue $k$. This linear map is self-adjoint hence diagonalizable in an orthogonal basis, and the diagonal form is a multiple of the identity. It is easily checked that the matrix remains a multiple of the identity in any basis of the tangent space, in particular in the basis $\left(\Phi_{u}, \Phi_{v}\right)$, which implies condition 2 .

\subsection{Principal directions.}

Let us focus on the maximum principal direction $d_{1}$. A vector of direction $d_{1}$ is an eigenvector of $W$ for the eigenvalue $k_{1}$. Denote:

$$
W-k_{1} I d=\left(\begin{array}{cc}
w_{11}-k_{1} & w_{12} \\
w_{21} & w_{22}-k_{1}
\end{array}\right) .
$$

At non umbilic points, the matrix $W-k_{1} I d$ has rank one, hence either $\left(-w_{12}, w_{11}-k_{1}\right) \neq(0,0)$ or $\left(-w_{22}+\right.$ $\left.k_{1}, w_{21}\right) \neq(0,0)$. Using the expression of $W$ given by Eq. (3), up to a normalization factor of $(\operatorname{det} I)^{3 / 2}$, a non zero maximal principal vector can be chosen as either

$v_{1}=2(\operatorname{det} I)^{3 / 2}\left(-w_{12}, w_{11}-k_{1}\right)=\left(-2 B, A-D-\sqrt{p_{2}}\right)$ or $w_{1}=2(\operatorname{det} I)^{3 / 2}\left(-w_{22}+k_{1}, w_{21}\right)=\left(A-D+\sqrt{p_{2}}, 2 C\right)$.

For the minimal principal direction $d_{2}$ one chooses $v_{2}=\left(-2 B, A-D+\sqrt{p_{2}}\right)$ and $w_{2}=\left(A-D-\sqrt{p_{2}}, 2 C\right)$.

Lemma. 2 One has the following relations:

$v_{1}=(0,0) \Leftrightarrow(B=0$ and $A \geq D)$,

$v_{2}=(0,0) \Leftrightarrow(B=0$ and $A \leq D)$,

$w_{1}=(0,0) \Leftrightarrow(C=0$ and $A \leq D)$,

$w_{2}=(0,0) \Leftrightarrow(C=0$ and $A \geq D)$.

Proof. The proofs being equivalent, we focus on the first one. One has:

$$
v_{1}=(0,0) \Leftrightarrow\left\{\begin{array} { l } 
{ B = 0 } \\
{ A - D = \sqrt { ( A - D ) ^ { 2 } + 4 B C } }
\end{array} \Leftrightarrow \left\{\begin{array} { l } 
{ B = 0 } \\
{ A - D = \sqrt { ( A - D ) ^ { 2 } } }
\end{array} \Leftrightarrow \left\{\begin{array}{l}
B=0 \\
A-D \geq 0
\end{array}\right.\right.\right.
$$

A direct consequence of lemma 2 is the following:

Observation. 21 . The two vector fields $v_{1}$ and $w_{1}$ vanish simultaneously exactly at umbilics. The same holds for $v_{2}$ and $w_{2}$.

2. The equation $\left\{v_{1}=(0,0)\right.$ or $\left.v_{2}=(0,0)\right\}$ is equivalent to $B=0$.

\section{Implicitly defining ridges}

In this section, we derive the implicit expression $P=0$ coding all ridges and umbilics. Before diving into the technicalities, we first outline the method. 


\subsection{Problem}

In characterizing ridges, a first difficulty comes from the fact that the sign of an extremality coefficient $\left(b_{0}\right.$ or $\left.b_{3}\right)$ is not well defined. Away from umbilics there are two unit opposite vectors $y_{1}$ and $-y_{1}$ orienting $d_{1}$. That is, one can define two extremality coefficients $b_{0}\left(y_{1}\right)=\left\langle d k_{1}, y_{1}\right\rangle$ and $b_{0}\left(-y_{1}\right)=\left\langle d k_{1},-y_{1}\right\rangle=-b_{0}\left(y_{1}\right)$. In addition, due to the presence of umbilics, there does not exist a continuous and non vanishing vector field defined on the surface except at umbilics such that its direction is the principal direction everywhere (in other words, umbilics are non orientable singularities of the principal direction fields). Therefore, the sign of $b_{0}$ cannot be globally defined on a surface with umbilics. Notice however the equation $b_{0}=0$ is not ambiguous. A second difficulty comes from umbilics where $b_{0}$ is not defined since $k_{1}$ is not smooth — that is $d k_{1}$ is not defined.

\subsection{Method outline}

Principal curvatures and directions read from the Weingarten map of the surface. At each point which is not an umbilic, one can define two vector fields $v_{1}$ or $w_{1}$ which are collinear with $d_{1}$, with the additional property that one (at least) of these two vectors is non vanishing. Let $z$ stand for one of these non vanishing vectors. The nullity of $b_{0}=\left\langle d k_{1}, y_{1}\right\rangle$ is equivalent to that of $\left\langle d k_{1}, z\right\rangle$ - that is the normalization of the vector along which the directional derivative is computed does not matter.

Using $v_{1}$ and $w_{1}$, the principal maximal vectors defined in the previous section, we obtain two independent equations of blue ridges. Each has the drawback of encoding, in addition to blue ridge points, the points where $v_{1}$ (or $w_{1}$ ) vanishes. As a consequence of observation 2, the conjunction of these two equations defines the set of blue ridges union the set of umbilics. The same holds for red ridges and the minimal principal vector fields $v_{2}$ and $w_{2}$. One has to note the symmetry between the equations for blue and red ridges in lemma 3. Eventually, combining the equation for blue ridges with $v_{1}$ and the equation for red ridges with $v_{2}$ gives the union of blue ridges, red ridges and the set of zeros of $v_{1}=0$ or $v_{2}=0$. This last set is also characterized by $B=0$ (observation 2), hence dividing by $B$ allows to eradicate these spurious points and yields the equation $P=0$ of blue and red ridges together with umbilics. One can think of this equation as an improved version of the Gaussian extremality $E_{g}=b_{0} b_{3}$ defined in [Thi96].

Our strategy cumulates several advantages: (i)blue and red ridges are processed at once, and the information is encoded in a single equation (ii)orientation issues arising when one is tracking the zero crossings of $b_{0}$ or $b_{3}$ by sign changes disappear. The only drawback is that one looses the color of the ridge. But this color is recovered with the evaluation of the sign of factors of the expression $P$.

\subsection{Implicit equation of ridges}

Lemma. 3 For a regular surface, there exist differentiable functions $a, a^{\prime}, b, b^{\prime}$ which are polynomials w.r.t. $A, B, C, D$ and $\operatorname{det} I$, as well as their first derivatives, such that:

1. the union of blue ridges and the set $\left\{v_{1}=0\right\}$ has equation $a \sqrt{p_{2}}+b=0$,

2. the union of blue ridges and the set $\left\{w_{1}=0\right\}$ has equation $a^{\prime} \sqrt{p_{2}}+b^{\prime}=0$,

3. the union of blue ridges and umbilics has equation $\left\{\begin{array}{l}a \sqrt{p_{2}}+b=0 \\ a^{\prime} \sqrt{p_{2}}+b^{\prime}=0\end{array}\right.$

4. the union of red ridges and the set $\left\{v_{2}=0\right\}$ has equation $a \sqrt{p_{2}}-b=0$,

5. the union of red ridges and the set $\left\{w_{2}=0\right\}$ has equation $a^{\prime} \sqrt{p_{2}}-b^{\prime}=0$,

6. the union of red ridges and umbilics has equation $\left\{\begin{array}{l}a \sqrt{p_{2}}-b=0 \\ a^{\prime} \sqrt{p_{2}}-b^{\prime}=0\end{array}\right.$

Moreover, $a, a^{\prime}, b, b^{\prime}$ are defined by the equations:

$$
a \sqrt{p_{2}}+b=\sqrt{p_{2}}(\operatorname{det} I)^{5 / 2}\left\langle d k_{1}, v_{1}\right\rangle \quad a^{\prime} \sqrt{p_{2}}+b^{\prime}=\sqrt{p_{2}}(\operatorname{det} I)^{5 / 2}\left\langle d k_{1}, w_{1}\right\rangle .
$$


Proof. The principal curvatures are not differentiable at umbilics, hence the equation $\left\langle d k_{1}, v_{1}\right\rangle$ is not defined at umbilics. On the other hand, since $\sqrt{p_{2}}$ only vanishes at umbilics and $\operatorname{det} I$ never vanishes, the equation $\sqrt{p_{2}}(\operatorname{det} I)^{5 / 2}\left\langle d k_{1}, v_{1}\right\rangle$ defines the same set. In addition, this equation is also well defined at umbilics. Rewritten as $a \sqrt{p_{2}}+b$, the functions $a$ and $b$ are then polynomial functions of the derivatives of the parameterization $\Phi$ up to the third order. The explicit expressions of $a$ and $b$ are given in [CFPR]. This equation describes the set of blue ridge points union the set where $v_{1}$ vanishes. A similar derivation yields the second claim. Finally, the third claim follows from observation 2 .

Results for red ridges are similar and the reader is referred to [CFPR] for the details.

Lemma. 4 1. If $p_{2}=0$ then $a=b=a^{\prime}=b^{\prime}=0$.

2. The set of purple points has equation $\left\{\begin{array}{l}a=b=a^{\prime}=b^{\prime}=0 \\ p_{2} \neq 0\end{array}\right.$

Proof. 1. If $p_{2}=0$, one has $A=D$ and $B=C=0$. Substituting these conditions in the expressions of $a, a^{\prime}, b, b^{\prime}$ gives the result, computations are sketched in [CFPR].

2. Let $p$ be a purple point, it is a ridge point and hence not an umbilic, then $p_{2} \neq 0$. The point $p$ is a blue and a red ridge point, hence it satisfies all equations of lemma 3. If $a \neq 0$ then equations 1 . and 4 . imply $\sqrt{p_{2}}=-b / a=b / a$ hence $b=0$ and $\sqrt{p_{2}}=0$ which is a contradiction. Consequently, $a=0$ and again equation 1. implies $b=0$. A similar argument with equation 2. and 5. gives $a^{\prime}=b^{\prime}=0$.

The converse is trivial: if $a=b=a^{\prime}=b^{\prime}=0$ then equations 3. and 6. imply that the point is a purple point or an umbilic. The additional condition $p_{2} \neq 0$ excludes umbilics.

The following definition is a technical tool to state the next theorem in a simple way. The meaning of the

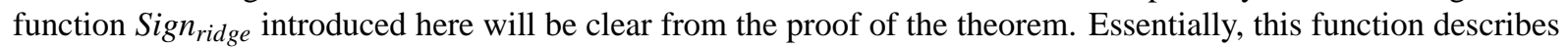
all the possible sign configurations for $a b$ and $a^{\prime} b^{\prime}$ at a ridge point.

Definition. 2 The function Sign $n_{\text {ridge }}$ takes the values

$$
\begin{aligned}
& -1 \text { if }\left\{\begin{array} { l } 
{ a b < 0 } \\
{ a ^ { \prime } b ^ { \prime } \leq 0 }
\end{array} \quad \text { or } \left\{\begin{array}{l}
a b \leq 0 \\
a^{\prime} b^{\prime}<0
\end{array},\right.\right. \\
& +1 \text { if }\left\{\begin{array} { l } 
{ a b > 0 } \\
{ a ^ { \prime } b ^ { \prime } \geq 0 }
\end{array} \quad \text { or } \left\{\begin{array}{l}
a b \geq 0 \\
a^{\prime} b^{\prime}>0
\end{array},\right.\right. \\
& 0 \text { if } a b=a^{\prime} b^{\prime}=0 .
\end{aligned}
$$

Theorem. 2 The union of blue ridges, red ridges and umbilics has equation $P=0$ with $P=\left(a^{2} p_{2}-b^{2}\right) / B$, and one also has $P=-\left(a^{\prime 2} p_{2}-b^{\prime 2}\right) / C=2\left(a^{\prime} b-a b^{\prime}\right)$. For a point of this set $\mathscr{P}$, one has:

- If $p_{2}=0$, the point is an umbilic.

- If $p_{2} \neq 0$ then:

- if Sign ${ }_{\text {ridge }}=-1$ then the point is a blue ridge point,

- if Sign $_{\text {ridge }}=+1$ then the point is a red ridge point,

- if Sign $_{\text {ridge }}=0$ then the point is a purple point.

Proof. To form the equation of $\mathscr{P}$, following the characterization of red and blue ridges in lemma 3 , and the vanishing of the vector fields $v_{1}$ and $v_{2}$ in lemma 2, we take the product of equations 1 . and 3 . of lemma 3 . The equivalence between the three equations of $\mathscr{P}$ is proved with the help of Maple, see [CFPR].

To qualify points on $\mathscr{P}$, first observe that the case $p_{2}=0$ has already been considered in lemma 4 . Therefore, assume $p_{2} \neq 0$, and first notice the following two simple facts:

- The equation $\left(a^{2} p_{2}-b^{2}\right) / B=0$ for $\mathscr{P}$ implies that $a=0 \Leftrightarrow b=0 \Leftrightarrow a b=0$. Similarly, the equation $-\left(a^{\prime 2} p_{2}-b^{\prime 2}\right) / C=0$ for $\mathscr{P}$ implies that $a^{\prime}=0 \Leftrightarrow b^{\prime}=0 \Leftrightarrow a^{\prime} b^{\prime}=0$. 
- If $a b \neq 0$ and $a^{\prime} b^{\prime} \neq 0$, the equation $a b^{\prime}-a^{\prime} b=0$ for $\mathscr{P}$ implies $b / a=b^{\prime} / a^{\prime}$, that is the signs of $a b$ and $a^{\prime} b^{\prime}$ agree.

These two facts explain the introduction of the function Sign $_{\text {ridge }}$ of definition 2. This function enumerates all disjoint possible configurations of signs for $a b$ and $a^{\prime} b^{\prime}$ for a point on $\mathscr{P}$. One can now study the different cases w.r.t. the signs of $a b$ and $a^{\prime} b^{\prime}$ or equivalently the values of the function Sign ridge.

Assume Sign $n_{\text {ridge }}=-1$.

-First case: $a b<0$. The equation $\left(a^{2} p_{2}-b^{2}\right) / 2 B=0$ implies that $\left(a \sqrt{p_{2}}+b\right)\left(a \sqrt{p_{2}}-b\right)=0$. Since $\sqrt{p_{2}}>0$, one must have $a \sqrt{p_{2}}+b=0$ which is equation 1 of lemma 3. From the second simple fact, either $a^{\prime} b^{\prime}<0$ or $a^{\prime} b^{\prime}=0$.

- For the first sub-case $a^{\prime} b^{\prime}<0$, equation $\left(a^{\prime 2} p_{2}-b^{\prime 2}\right) / C=0$ implies $\left(a^{\prime} \sqrt{p_{2}}+b^{\prime}\right)\left(a^{\prime} \sqrt{p_{2}}-b^{\prime}\right)=0$. Since $\sqrt{p_{2}}>0$, one must have $a^{\prime} \sqrt{p_{2}}+b^{\prime}=0$ which is equation 2 of lemma 3 .

- For the second sub-case $a^{\prime} b^{\prime}=0$, one has $a^{\prime}=b^{\prime}=0$ and the equation 2 of lemma 3 is also satisfied. (Moreover, equation 5 is also satisfied which implies that $w_{2}=0$ ).

In both cases, equations 1 and 2 or equivalently equation 3 are satisfied. Since one has excluded umbilics, the point is a blue ridge point.

-Second case: $a b=0$. One has $a^{\prime} b^{\prime}<0$ the study is similar to the above. $a b=0$ implies equation 1 and $a^{\prime} b^{\prime}<0$ implies equation 2 of lemma 3 . The point is a blue ridge point.

Assume Sign ridge $_{1}=1$.

This case is the exact symmetric of the previous, one only has to exchange the roles of $a, b$ and $a^{\prime}, b^{\prime}$.

Assume Sign ridge $_{\text {e }}=0$.

The first simple fact implies $a=b=a^{\prime}=b^{\prime}=0$ and lemma 4 identifies a purple point.

As shown along the proof, the conjunctions $<,=$ and $=,<$ in the definition of $\operatorname{Sign}_{\text {ridge }}=-1$ correspond to the blue ridge points where the vector fields $w_{2}$ and $v_{2}$ vanish. The same holds for Sign ridge $=1$ and $w_{1}$ and $v_{1}$. One can also observe that the basic ingredient of the previous proof is to transform an equation with a square root into a system with an inequality. More formally:

Observation. 3 For $x, y, z$ real numbers and $z \geq 0$, one has:

$$
x \sqrt{z}+y=0 \Longleftrightarrow\left\{\begin{array}{l}
x^{2} z-y^{2}=0 \\
x y \leq 0
\end{array}\right.
$$

\subsection{Singular points of $\mathscr{P}$}

Having characterized umbilics, purple points and ridges in the domain $\mathscr{D}$ with implicit equations, an interesting question is to relate the properties of these equations to the classical differential geometric properties of these points.

In particular, recall that generically (with the description of surfaces with contact theory [ $\left.\mathrm{HGY}^{+} 99\right]$ ), umbilics of a surface are either 1-ridge umbilics or 3-ridge umbilics. This means that there are either 1 or 3 non-singular ridge branches passing through an umbilic. The later are obviously singular points of $\mathscr{P}$ since three branches of the curve are crossing at the umbilic. For the former ones, it is appealing to believe they are regular points since the tangent space to the ridge curve on the surface at such points is well defined and can be derived from the cubic

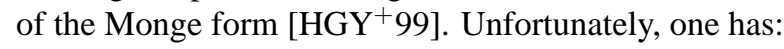

Theorem. 3 Umbilics are singular points of multiplicity at least 3 of the function $P$ (i.e. the gradient and the Hessian of $P$ vanish).

Proof. Following the notations of Porteous [Por01], denote $P_{k}, k=1, \ldots, 3$ the $k$ th times linear form associated with $P$, that is $P_{k}=\left[\partial P /\left(\partial u^{k-i} \partial v^{i}\right)\right]_{i=0, \ldots, k}$. Phrased differently, $P_{1}$ is the gradient, $P_{2}$ is the vector whose three entries encodes the Hessian of $P$, etc. To show that the multiplicity of an umbilic of coordinates $\left(u_{0}, v_{0}\right)$ is at least three, we need to show that $P_{1}\left(u_{0}, v_{0}\right)=[0,0], P_{2}\left(u_{0}, v_{0}\right)=[0,0,0]$. We naturally do not know the coordinates 
of umbilics, but lemma 1 provides the umbilical conditions. The proof consists of computing derivatives and performing the appropriate substitutions under Maple, and is given in [CFPR].

We can go one step further so as to relate the type of the cubic $P_{3}$ - the third derivative of $P$ - to the number of non-singular ridge branches at the umbilic.

Theorem. 4 The classification of an umbilic as 1-ridge of 3-ridges from $P_{3}$ goes as follows:

- If $P_{3}$ is elliptic, that is the discriminant of $P_{3}$ is positive $\left(\delta\left(P_{3}\right)>0\right)$, then the umbilic is a 3-ridge umbilic and the 3 tangent lines to the ridges at the umbilic are distinct.

- If $P_{3}$ is hyperbolic $\left(\delta\left(P_{3}\right)<0\right)$ then the umbilic is a 1-ridge umbilic.

Proof. Since the properties of interest here are local ones, studying ridges on the surface or in the parametric domain is equivalent because the parameterization is a local diffeomorphism. More precisely the parameterization $\Phi$ maps a curve passing through $\left(u_{0}, v_{0}\right) \in \mathscr{D}$ to a curve passing through the umbilic $p_{0}=\Phi\left(u_{0}, v_{0}\right)$ on the surface $S=\Phi(\mathscr{D})$. Moreover, the invertible linear map $d \Phi_{\left(u_{0}, v_{0}\right)}$ maps the tangent to the curve in $\mathscr{D}$ at $\left(u_{0}, v_{0}\right)$ to the tangent at $p_{0}$ to its image curve in the tangent space $T_{p_{0}} S$.

Having observed the multiplicity of umbilics is at least three, we resort to singularity theory. From [AGZV85, Section 11.2, p157], we know that a cubic whose discriminant is non null is equivalent up to a linear transformation to the normal form $y\left(x^{2} \pm y^{2}\right)$. Moreover, a function having a vanishing second order Taylor expansion and its third derivative of this form is diffeomorphic to the same normal form. Therefore, whenever the discriminant of $P_{3}$ is non null, up to a diffeomorphism, the umbilic is a so called $D_{4}^{ \pm}$singularity of $P$, whose normal form is $y\left(x^{2} \pm y^{2}\right)$. It is then easily seen that the zero level set consists of three non-singular curves through the umbilic with distinct tangents which are the factor lines of the cubic. For a $D_{4}^{-}$singularity $\left(\delta\left(P_{3}\right)>0\right)$, these 3 curves are real curves and the umbilic is a 3 -ridge. For a $D_{4}^{+}$singularity $\left.\delta\left(P_{3}\right)<0\right)$, only one curve is real and the umbilic is a 1-ridge.

Note that the classifications of umbilics with the Monge cubic $C_{M}$ and the cubic $P_{3}$ do not coincide. The Monge cubic $C_{M}$ is the third order part of the Taylor expansion of the surface parametrized by its tangent plane. Indeed if $C_{M}$ is elliptic, it may occur that two ridges have the same tangent. In such a case, the cubic $P_{3}$ is not elliptic since $\delta\left(P_{3}\right)=0$.

Since purple points correspond to the intersection of two ridges, one has:

Theorem. 5 Purple points are singular points of multiplicity at least 2 of the function $P$ (i.e. the gradient of $P$ vanishes).

Proof. It follows from the equation $P=2\left(a^{\prime} b-a b^{\prime}\right)$ that $d P=2\left(d\left(a^{\prime}\right) b+a^{\prime} d(b)-d(a) b-a d(b)\right)$. At purple points one has $a=a^{\prime}=b=b^{\prime}=0$ hence $d P=0$.

\section{Implicit system for turning points and ridge type}

In this section, we define a system of equations that encodes turning points. Once these turning points identified, we show how to retrieve the type (elliptic or hyperbolic) of a ridge from a sign evaluation.

\subsection{Problem}

Going one step further in the description of ridges requires distinguishing between ridges which are maxima or minima of the principal curvatures. Following the classical terminology [Por01, $\mathrm{HGY}^{+}$99], a blue (red) ridge changes from a maximum to a minimum at a blue (red) turning point. These turning points are witnessed by the vanishing of the second derivative of the principal curvature along its curvature line. Since we are working from a parameterization, denoting Hess the Hessian matrix of either principal curvature, we have:

Observation. 4 A blue turning point is a blue ridge point where $d_{1}^{T} H e s s\left(k_{1}\right) d_{1}=0$. Similarly, a red turning point is a red ridge point with $d_{2}^{T} \operatorname{Hess}\left(k_{2}\right) d_{2}=0$. 
Generically, turning points are not purple points, however we shall provide conditions identifying these cases. Even less generic is the existence of a purple point which is also a blue and a red turning point, a situation for which we also provide conditions.

Once turning points have been found, reporting elliptic and hyperbolic ridge sections is especially easy. For ridges through umbilics, since ridges at umbilics are hyperbolic, and the two types alternate at turning points, the task is immediate. For ridges avoiding umbilics, one just has to test the sign of $d_{1}^{T} \operatorname{Hess}\left(k_{1}\right) d_{1}$ or $d_{2}^{T} \operatorname{Hess}\left(k_{2}\right) d_{2}$ at a ridge points, and then propagate the alternation at turning points.

\subsection{Method outline}

We focus on blue turning points since the method for red turning points is similar. As already pointed out, we do not have a global expression of the blue direction $d_{1}$, but only the two blue vector fields $v_{1}$ and $w_{1}$ vanishing on some curves going through umbilics. Consequently, we have to combine equations with these two fields to get a global expression of turning points. A blue ridge point is a blue turning point iff $d_{1}^{T} \operatorname{Hess}\left(k_{1}\right) d_{1}=0$. This last equation is equivalent to $p_{2}^{3 / 2}(\operatorname{det} I)^{7 / 2} v_{1}^{T} \operatorname{Hess}\left(k_{1}\right) v_{1}=0$ when the vector field $v_{1}$ does not vanish. This equation is in addition well defined at umbilics. The same holds for the equation $p_{2}^{3 / 2}(\operatorname{det} I)^{7 / 2} w_{1}^{T} \operatorname{Hess}\left(k_{1}\right) w_{1}=0$ and the solutions of $w_{1}=(0,0)$. As a consequence of observation 2 , the conjunction of these two equations defines the set of blue turning points.

The drawback of distinguishing the color of the turning points is that equations contain a square root. Combining the equations for blue and red turning points gives an equation $Q=0$ without square roots. The intersection of the corresponding curve $\mathscr{Q}$ with the ridge curve $\mathscr{P}$ and sign evaluations allow to retrieve all turning points and their color.

\subsection{System for turning points}

Lemma. 5 For a regular surface, there exist differentiable functions $\alpha, \alpha^{\prime}, \beta, \beta^{\prime}$ which are polynomials w.r.t. $A, B, C, D$ and $\operatorname{det} I$, as well as their first and second derivatives, such that:

1. $p_{2}^{3 / 2}(\operatorname{det} I)^{7 / 2} v_{1}^{T} \operatorname{Hess}\left(k_{1}\right) v_{1}=\alpha \sqrt{p_{2}}+\beta$.

2. $p_{2}^{3 / 2}(\operatorname{det} I)^{7 / 2} w_{1}^{T} \operatorname{Hess}\left(k_{1}\right) w_{1}=\alpha^{\prime} \sqrt{p_{2}}+\beta^{\prime}$.

3. A blue ridge point is a blue turning point iff $\left\{\begin{array}{l}\alpha \sqrt{p_{2}}+\beta=0 \\ \alpha^{\prime} \sqrt{p_{2}}+\beta^{\prime}=0\end{array}\right.$

4. $p_{2}^{3 / 2}(\operatorname{det} I)^{7 / 2} v_{2}^{T} \operatorname{Hess}\left(k_{2}\right) v_{2}=\alpha \sqrt{p_{2}}-\beta$.

5. $p_{2}^{3 / 2}(\operatorname{det} I)^{7 / 2} w_{2}^{T} \operatorname{Hess}\left(k_{2}\right) w_{2}=\alpha^{\prime} \sqrt{p_{2}}-\beta^{\prime}$.

6. A red ridge point is a red turning point iff $\left\{\begin{array}{l}\alpha \sqrt{p_{2}}-\beta=0 \\ \alpha^{\prime} \sqrt{p_{2}}-\beta^{\prime}=0\end{array}\right.$

Proof. Calculations for points 1-2-4-5 are performed with Maple cf. [CFPR]. Blue turning points are blue ridge points on $\mathscr{P}$ where $v_{1}^{T} \operatorname{Hess}\left(k_{1}\right) v_{1}=0$. This equation is not defined at umbilics where principal curvatures are not differentiable. Nevertheless, including umbilics and points where $v_{1}$ vanishes, this equation is equivalent to $p_{2}^{3 / 2}(\operatorname{det} I)^{7 / 2} v_{1}^{T} \operatorname{Hess}\left(k_{1}\right) v_{1}=0$. This equation is rewritten as $\alpha \sqrt{p_{2}}+\beta=0$ and yields point 1 . The same analysis holds for $w_{1}$ and yields point 2. Point 3. is a consequence of observation 2. Results for red turning points are similar and the reader is referred to [CFPR] for the details.

The following definition is a technical tool to state the next theorem in a simple way. As we shall see along the proof, this function describes all the possible sign configurations for $\alpha \beta$ and $\alpha^{\prime} \beta^{\prime}$ at a turning point.

Definition. 3 The function Sign turn takes the values 


$$
\begin{aligned}
& -1 \text { if }\left\{\begin{array} { l } 
{ \alpha \beta < 0 } \\
{ \alpha ^ { \prime } \beta ^ { \prime } \leq 0 }
\end{array} \quad \text { or } \left\{\begin{array}{l}
\alpha \beta \leq 0 \\
\alpha^{\prime} \beta^{\prime}<0
\end{array},\right.\right. \\
& +1 \text { if }\left\{\begin{array} { l } 
{ \alpha \beta > 0 } \\
{ \alpha ^ { \prime } \beta ^ { \prime } \geq 0 }
\end{array} \text { or } \left\{\begin{array}{l}
\alpha \beta \geq 0 \\
\alpha^{\prime} \beta^{\prime}>0
\end{array},\right.\right. \\
& \text { o if } \alpha \beta=\alpha^{\prime} \beta^{\prime}=0 .
\end{aligned}
$$

Theorem. 6 Let $Q$ be the smooth function which is a polynomial w.r.t. A,B,C,D and $\operatorname{det} I$, as well as their first and second derivatives defined by

$$
Q=\left(\alpha^{2} p_{2}-\beta^{2}\right) / B^{2}=\left(\alpha^{\prime 2} p_{2}-\beta^{\prime 2}\right) / C^{2}=2\left(\alpha^{\prime} \beta-\alpha \beta^{\prime}\right) /(D-A) .
$$

The system $\left\{\begin{array}{l}P=0 \\ Q=0\end{array} \quad\right.$ encodes turning points in the following sense. For a point, solution of this system, one has:

- If $p_{2}=0$, the point is an umbilic.

- If $p_{2} \neq 0$ then:

- if Sign ridge $_{1}=-1$ and Sign $n_{\text {turn }} \leq 0$ then the point is a blue turning point,

- if Sign ridge $=+1$ and Sign turn $\geq 0$ then the point is a red turning point,

- if Sign ridge $=0$ then the point is purple point and in addition

$*$ if Sign $_{\text {turn }}=-1$ then the point is also a blue turning point,

$*$ if Sign $_{\text {turn }}=+1$ then the point is also a red turning point,

$*$ if Sign $n_{\text {turn }}=0$ then the point is also a blue and a red turning point.

Proof. Following lemma 5, we form the equation of $Q$ by taking the products of 1 . and 4 . in the lemma. Equalities of equation (9) are performed with Maple cf. [CFPR].

The case $p_{2}=0$ has already been considered in lemma 4 . Assume that $p_{2} \neq 0$, and first notice the following two simple facts:

- The equation $\left(\alpha^{2} p_{2}-\beta^{2}\right) / B^{2}=0$ for $\mathscr{Q}$ implies that $\alpha=0 \Leftrightarrow \beta=0 \Leftrightarrow \alpha \beta=0$. Similarly, the equation $\left(\alpha^{\prime 2} p_{2}-\beta^{\prime 2}\right) / C^{2}=0$ for $\mathscr{Q}$ implies that $\alpha^{\prime}=0 \Leftrightarrow \beta^{\prime}=0 \Leftrightarrow \alpha^{\prime} \beta^{\prime}=0$.

- If $\alpha \beta \neq 0$ and $\alpha^{\prime} \beta^{\prime} \neq 0$, the equation $2\left(\alpha^{\prime} \beta-\alpha \beta^{\prime}\right) /(D-A)=0$ for $\mathscr{Q}$ implies $\beta / \alpha=\beta^{\prime} / \alpha^{\prime}$, that is the signs of $\alpha \beta$ and $\alpha^{\prime} \beta^{\prime}$ agree.

These two facts explain the introduction of the function $\operatorname{Sign}_{\text {turn }}$ of definition 3. This function enumerates all disjoint possible configurations of signs for $\alpha \beta$ and $\alpha^{\prime} \beta^{\prime}$ for a point on $\mathscr{Q}$. The analysis of the different cases is similar to that of the proof of theorem 2 , the basic ingredient being observation 3 .

Observation. 5 Note that in the formulation of equation (9) there are solutions of the system $(P=0$ and $Q=0)$ which are not turning points nor umbilics. These points are characterized by $\left(S_{i g n}\right.$ ridge $_{2}=-1$ and Sign $\left.n_{\text {turn }}=+1\right)$ or $\left(\right.$ Sign $_{\text {ridge }}=+1$ and Sign $\left.n_{\text {turn }}=-1\right)$. This drawback is unavoidable since equations avoiding the term $\sqrt{p_{2}}$ cannot distinguish colors.

Observation. 6 The following holds:

- $p_{2}=0$ implies $\alpha=\alpha^{\prime}=\beta=\beta^{\prime}=0$

- $\alpha=\alpha^{\prime}=\beta=\beta^{\prime}=0$ are singularities of $Q$ of multiplicity at least 2 .

To test if a blue ridge segment between two turning points is a maximum or a minimum requires the evaluation of the sign of $\alpha \sqrt{p_{2}}+\beta$ or $\alpha^{\prime} \sqrt{p_{2}}+\beta^{\prime}$, which cannot vanish simultaneously. 


\section{Polynomial surfaces}

A fundamental class of surfaces used in Computer Aided Geometric Design consist of Bezier surfaces and splines. In this section, we state some elementary observations on the objects studied so far, for the particular case of polynomial parametric surfaces. Notice that the parameterization can be general, in which case $\Phi(u, v)=$ $(x(u, v), y(u, v), z(u, v))$, or can be a height function $\Phi(u, v)=(u, v, z(u, v))$.

\subsection{About $W$ and the vector fields}

Using Eq. (3), we first observe that if $\Phi$ is a polynomial then the coefficients $A, B, C$ and $D$ are also polynomials - this explains the factor $(\operatorname{det} I)^{3 / 2}$ in the denominator of $W$ in equation (3). Thus, in the polynomial case, the equation of ridges is algebraic. Hence the set of all ridges and umbilics is globally described by an algebraic curve. The function $Q$ is also a polynomial so that turning points are described by a polynomial system.

An interesting corollary of lemma 2 for the case of polynomial surfaces is the following:

Observation. 7 Given a principal vector $v$, denote $Z_{v}$ the zero set of $v$ i.e. the set of points where $v$ vanishes. For a polynomial surface, the sets $Z_{v_{1}}, Z_{w_{1}}, Z_{v_{2}}, Z_{w_{2}}$ are semi-algebraic sets.

\subsection{Degrees of expressions}

As a corollary of Thm. 2 and 6, one can give upper bounds for the total degrees of expressions w.r.t. that of the parameterization. Distinguishing the cases where $\Phi$ is a general parameterization or a height function (that is $\Phi(u, v)=(u, v, h(u, v)))$ with $h(u, v)$ and denoting $d$ the total degree of $\Phi$, table 1 gives the total degrees of $A, B, C, D, \operatorname{det} I, P$ and $Q$.

Note that in the case of a height function, $P$ is divided by its factor $\operatorname{det} I^{2}$, and $Q$ is divided by its factor $\operatorname{det} I$ (cf. [CFPR]).

\begin{tabular}{|c|c|c|}
\hline Polynomials & General parameterization & Height function \\
\hline$A, B, C, D$ & $d_{1}=5 d-6$ & $d_{1_{h}}=3 d-4$ \\
\hline $\operatorname{det} I$ & $d_{2}=4 d-4$ & $d_{2}=4 d-4$ \\
\hline$P$ & $5 d_{1}+2 d_{2}-2=33 d-40$ & $5 d_{1_{h}}-2=15 d-22$ \\
\hline$Q$ & $10 d_{1}+4 d_{2}-4=66 d-80$ & $10 d_{1_{h}}+3 d_{2}-4=42 d-56$ \\
\hline
\end{tabular}

Figure 1: Total degrees of polynomials

\section{3 illustrations}

Theorem 2 is effective and allows one to report certified ridges of polynomial parametric surfaces without resorting to local orientation procedures. Moreover, results of section 6 enable to compute the ridge types.

Without engaging into the algebraic developments carried out in [CFPR05], we just provide illustrations of ridges for two surfaces defined over the domain $\mathscr{D}=[0,1] \times[0,1]$ by the functions $h 1$ and $h 2$

$$
\begin{array}{r}
h 1(u, v)=-1 / 12 u-\frac{35}{24} v+5 / 2 u v+1 / 2 u^{2} v-3 u v^{2}-\frac{17}{12} u^{2}+4 / 3 v^{2}+u^{3}+1 / 3 v^{3}+\frac{5}{16} \\
h 2(u, v)=\frac{36}{5} u^{3} v^{3}-\frac{126}{5} u^{3} v^{2}+18 u^{3} v-\frac{54}{5} u^{2} v^{3}+\frac{189}{5} u^{2} v^{2}-27 u^{2} v+\frac{18}{5} u v^{3}-\frac{63}{5} u v^{2}+9 u v
\end{array}
$$

The computations of the implicit curves have been performed using Maple 9.5, see [CFPR]. For $h 1$, the ridge curve is of total degree 19, has 210 terms and has one 3-ridge umbilic. For $h 2$, the ridge curve is of total degree 58, has 941 terms and has five 1-ridge umbilics. The surfaces and ridges with their types are displayed on Figs. 2 and 3. The thick (resp. thin) black lines are blue elliptic (resp. hyperbolic) ridges and the thick (resp. thin) grey lines are red elliptic (resp. hyperbolic) ridges. 


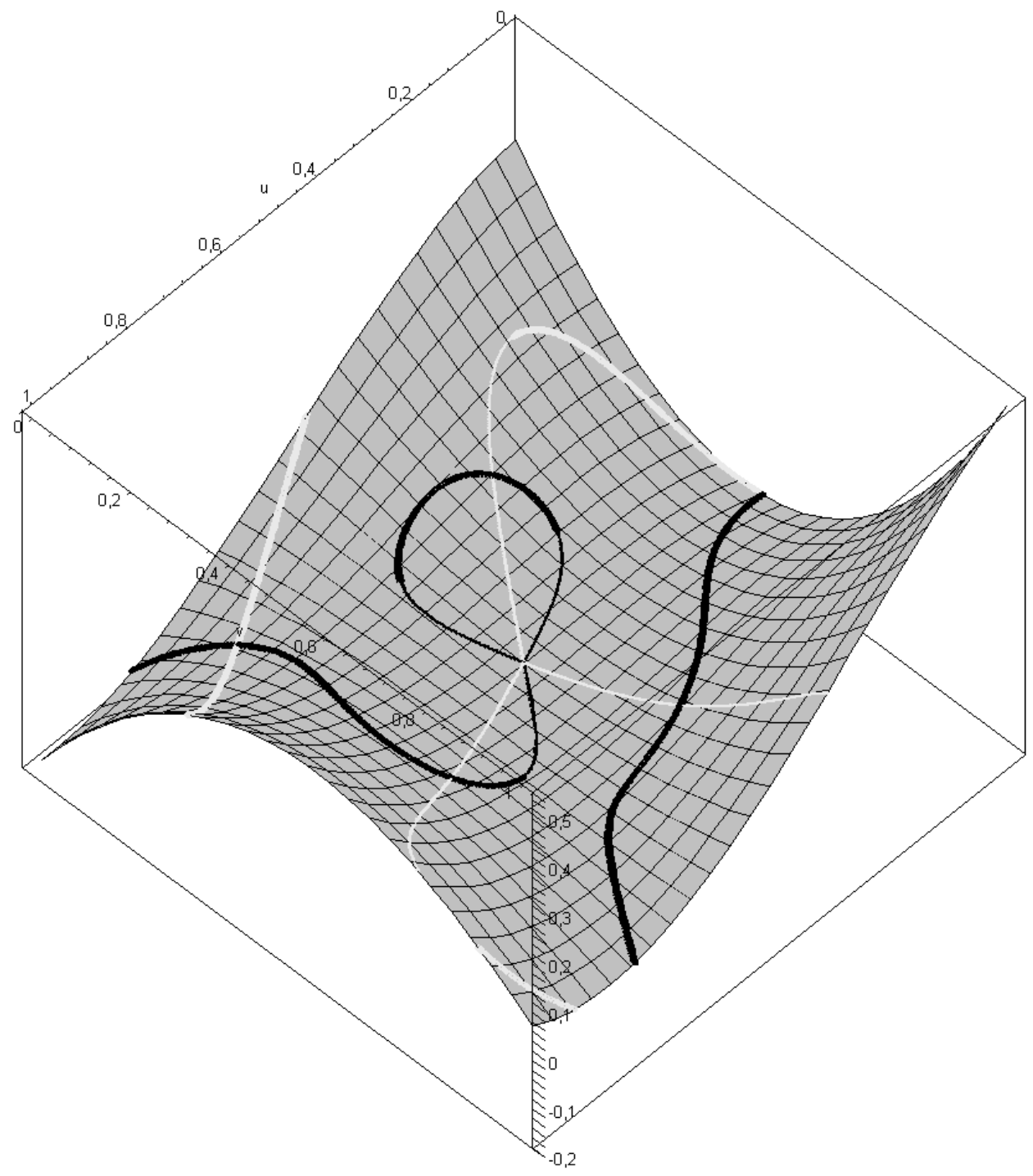

Figure 2: Ridges of the surface $h 1$ : thick (resp. thin) black lines are blue elliptic (resp. hyperbolic) ridges; thick (resp. thin) grey lines are red elliptic (resp. hyperbolic) ridges

\section{Conclusion}

This paper sets the implicit equation $P=0$ of the singular curve encoding all ridges and umbilics of a smooth parametric surface. From a mathematical standpoint, a corollary of this result shows that ridges and umbilics of polynomial surfaces are polynomial objects. From an algorithmic perspective, this result paves the alley for the development of certified algorithms reporting ridges without resorting to local orientation procedures. For algebraic surfaces, such algorithms are developed in a companion paper.

Acknowledgments. F. Cazals and M. Pouget acknowledge the support of the AIM@ Shape (IST-506766) and Algorithms for Complex Shapes (IST-006413) European projects.

\section{References}

[ABL03] D. Attali, J.-D. Boissonnat, and A. Lieutier. Complexity of the delaunay triangulation of points on surfaces the smooth case. In ACM SoCG, San Diego, 2003. 


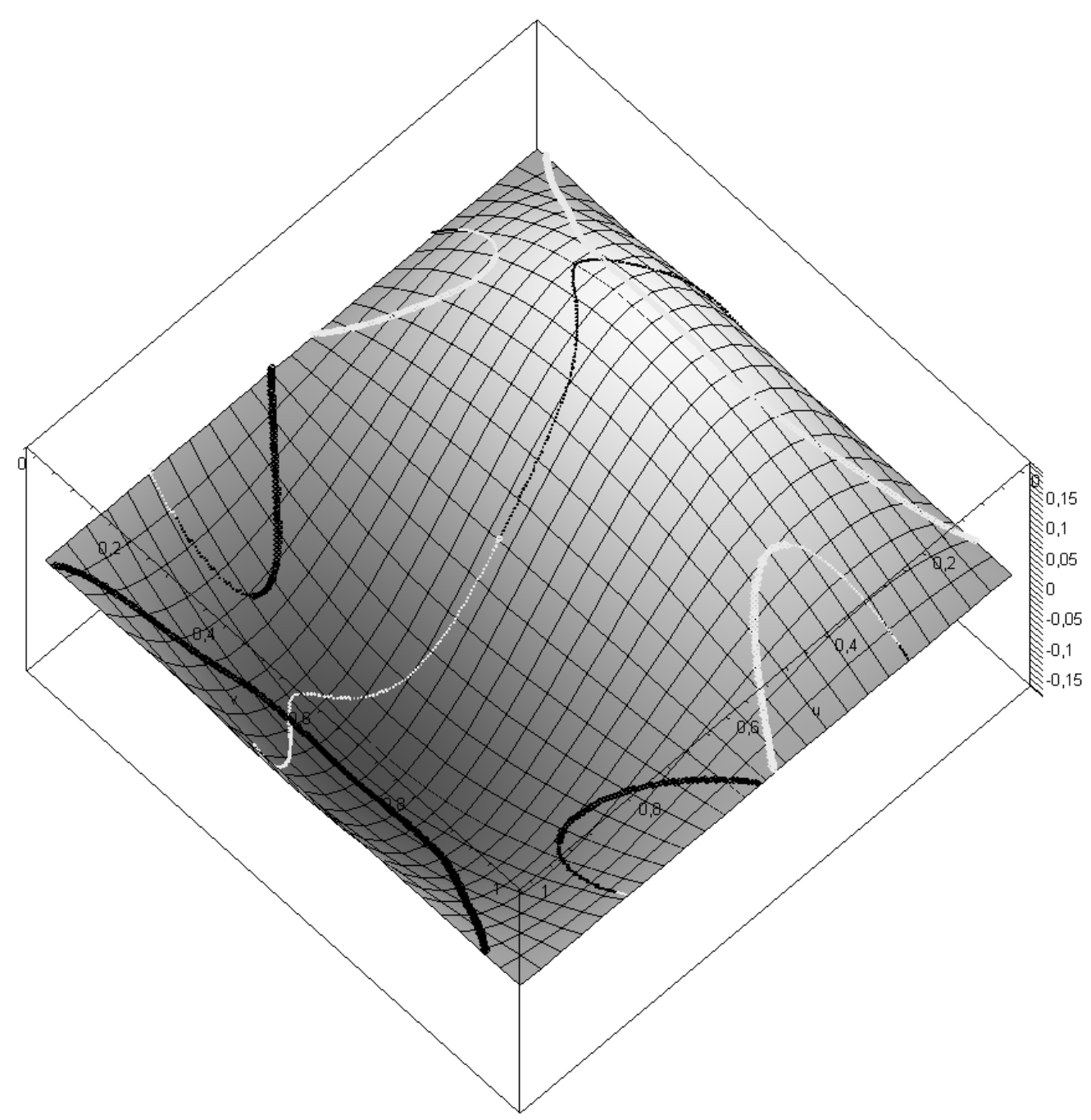

Figure 3: Ridges of the surface $h 2$ : thick (resp. thin) black lines are blue elliptic (resp. hyperbolic) ridges; thick (resp. thin) grey lines are red elliptic (resp. hyperbolic) ridges

[AGZV85] V. I. Arnold, S. M. Gusein-Zade, and A. N. Varchenko. Singularities of Differentiable Maps, volume 82 of Monographs in Mathematics. Birkhauser, Boston, MA, U.S.A., 1985. Vol. 1. The classification of critical points, caustics and wave fronts. Vol. 2, 1988.

[CFPR] F. Cazals, J.-C. Faugère, M. Pouget, and F. Rouillier. Maple worksheet. Technical report, ftp://ftpsop.inria.fr/geometrica/mpouget/ref-maple-ridge-turn.mw.

[CFPR05] F. Cazals, J.-C. Faugère, M. Pouget, and F. Rouillier. The implicit structure of ridges of a smooth parametric surface. Technical Report 5608, INRIA, 2005. Submitted.

[CP05a] F. Cazals and M. Pouget. Smooth surfaces, umbilics, lines of curvatures, foliations, ridges and the medial axis: selected topics. Int. J. of Computational Geometry and Applications, 15(5):511-536, 2005.

[CP05b] F. Cazals and M. Pouget. Topology driven algorithms for ridge extraction on meshes. Technical Report RR-5526, INRIA, 2005. 
[dC76] M. de Carmo. Differential Geometry of Curves and Surfaces. Prentice Hall, Englewood Cliffs, NJ, 1976.

[HCV52] D. Hilbert and S. Cohn-Vossen. Geometry and the Imagination. Chelsea, 1952.

$\left[\mathrm{HGY}^{+} 99\right]$ P. W. Hallinan, G. Gordon, A.L. Yuille, P. Giblin, and D. Mumford. Two-and Three-Dimensional Patterns of the Face. A.K.Peters, 1999.

[Koe90] J.J. Koenderink. Solid Shape. MIT, 1990.

[Mor90] R. Morris. Symmetry of Curves and the Geometry of Surfaces: two Explorations with the aid of Computer Graphics. Phd Thesis, 1990.

[Mor96] R. Morris. The sub-parabolic lines of a surface. In Glen Mullineux, editor, Mathematics of Surfaces VI, IMA new series 58, pages 79-102. Clarendon Press, Oxford, 1996.

[PAT00] X. Pennec, N. Ayache, and J.-P. Thirion. Landmark-based registration using features identified through differential geometry. In I. Bankman, editor, Handbook of Medical Imaging. Academic Press, 2000.

[Por71] I. Porteous. The normal singularities of a submanifold. J. Diff. Geom., 5, 1971.

[Por01] I. Porteous. Geometric Differentiation (2nd Edition). Cambridge University Press, 2001.

[Thi96] J.-P. Thirion. The extremal mesh and the understanding of $3 \mathrm{~d}$ surfaces. International Journal of Computer Vision, 19(2):115-128, August 1996. 


\section{Appendix : Maple calculations}

\section{PRINCIPAL DIRECTIONS, CURVATURES and DERIVATIVES}

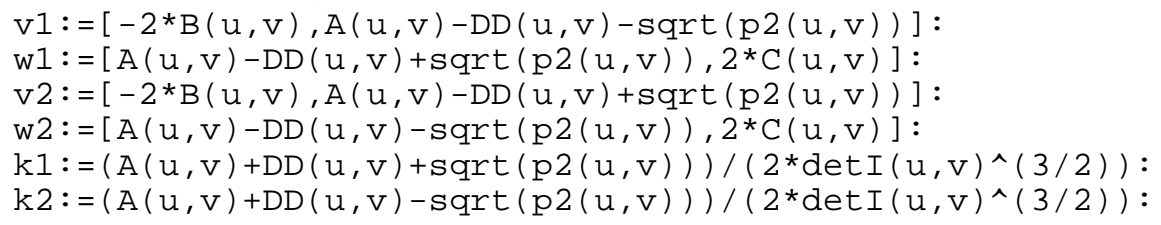

First derivatives

$>\operatorname{dk} 1:=[\operatorname{diff}(\mathrm{k} 1, \mathrm{u}), \operatorname{diff}(\mathrm{k} 1, \mathrm{v})]:$

$>\operatorname{dk} 2:=[\operatorname{diff}(\mathrm{k} 2, \mathrm{u}), \operatorname{diff}(\mathrm{k} 2, \mathrm{v})]:$

Second derivatives

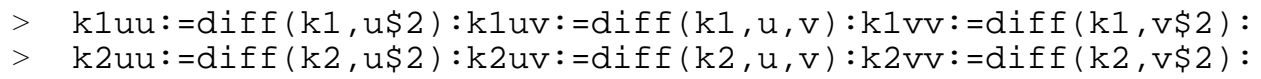

\section{RIDGES}

Blue and red equations wrt the vector fields v1, v2, w1, w2.

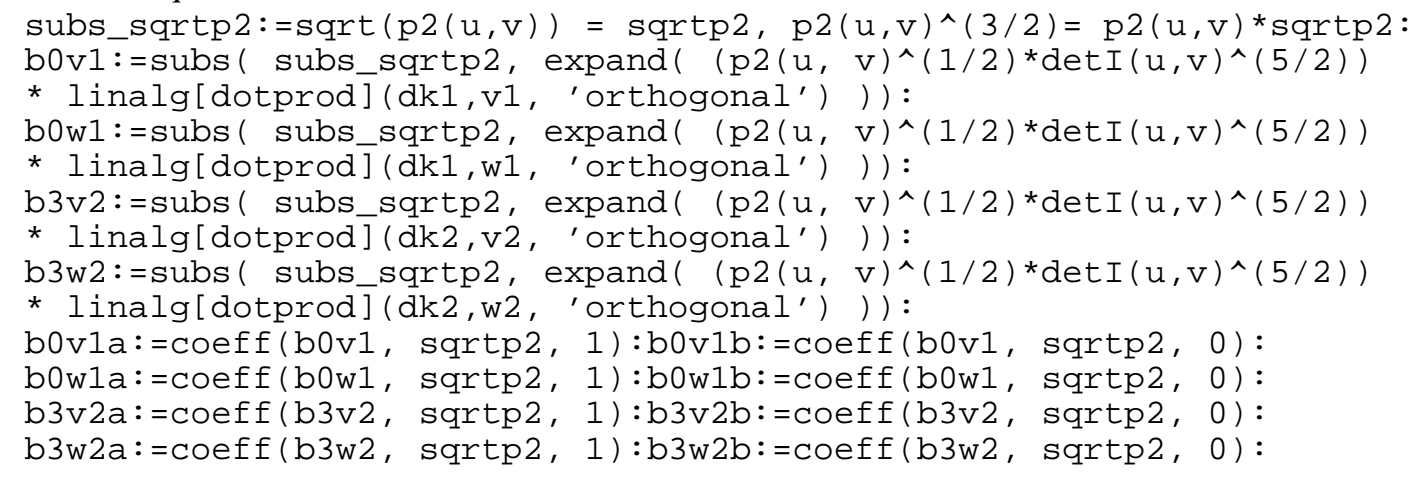

Identities between b0 with (v1,w1) and b3 with (v2,w2).

$>$ [b3v2a-b0v1a, b0v1b+b3v2b, b3w2a-b0w1a, b0w1b+b3w2b] ;

Definition of a,b,abis,bbis

$$
[0,0,0,0]
$$

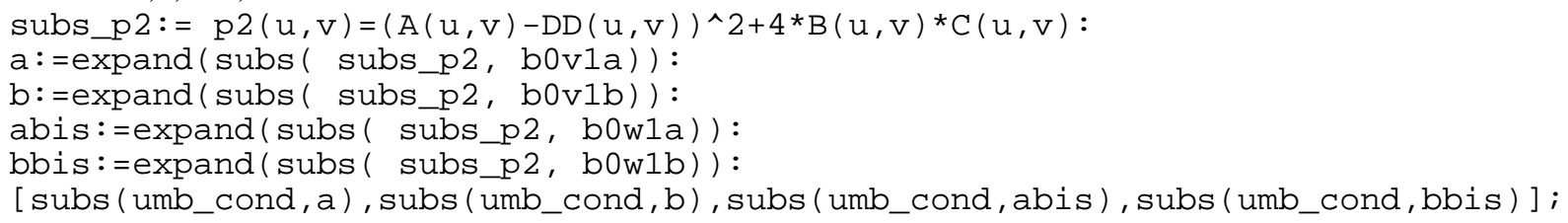

$$
[0,0,0,0]
$$

Ridge equation, identies

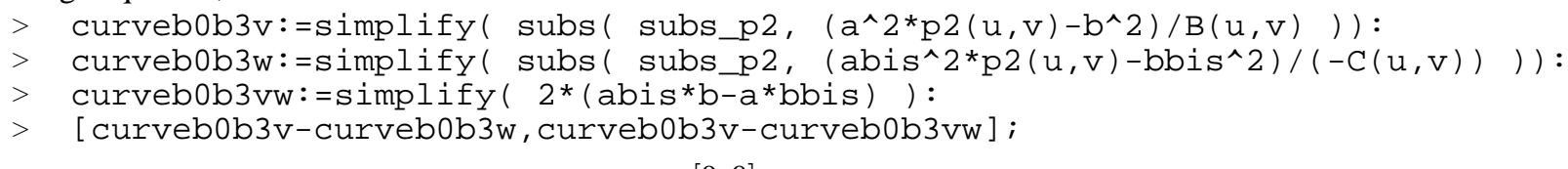

$$
[0,0]
$$

Final result: ridge has 170 terms of the form 5 times a term amongst A,B,C,DD and twice detI and 2 derivatives

$>$ ridge:=simplify (curveb0b3vw/content (curveb0b3vw)) :

$>\quad[$ whattype (ridge), nops (ridge), op (1, ridge) ];

Umbilics are points on $\mathrm{P}=0$ of multiplicity at least 3

$$
\left[{ }^{\prime}+{ }^{\prime}, 170,4 \operatorname{det} I(u, v)^{2} B(u, v)\left(\frac{\partial}{\partial u} A(u, v)\right)\left(\frac{\partial}{\partial v} C(u, v)\right) D D(u, v)^{2}\right]
$$




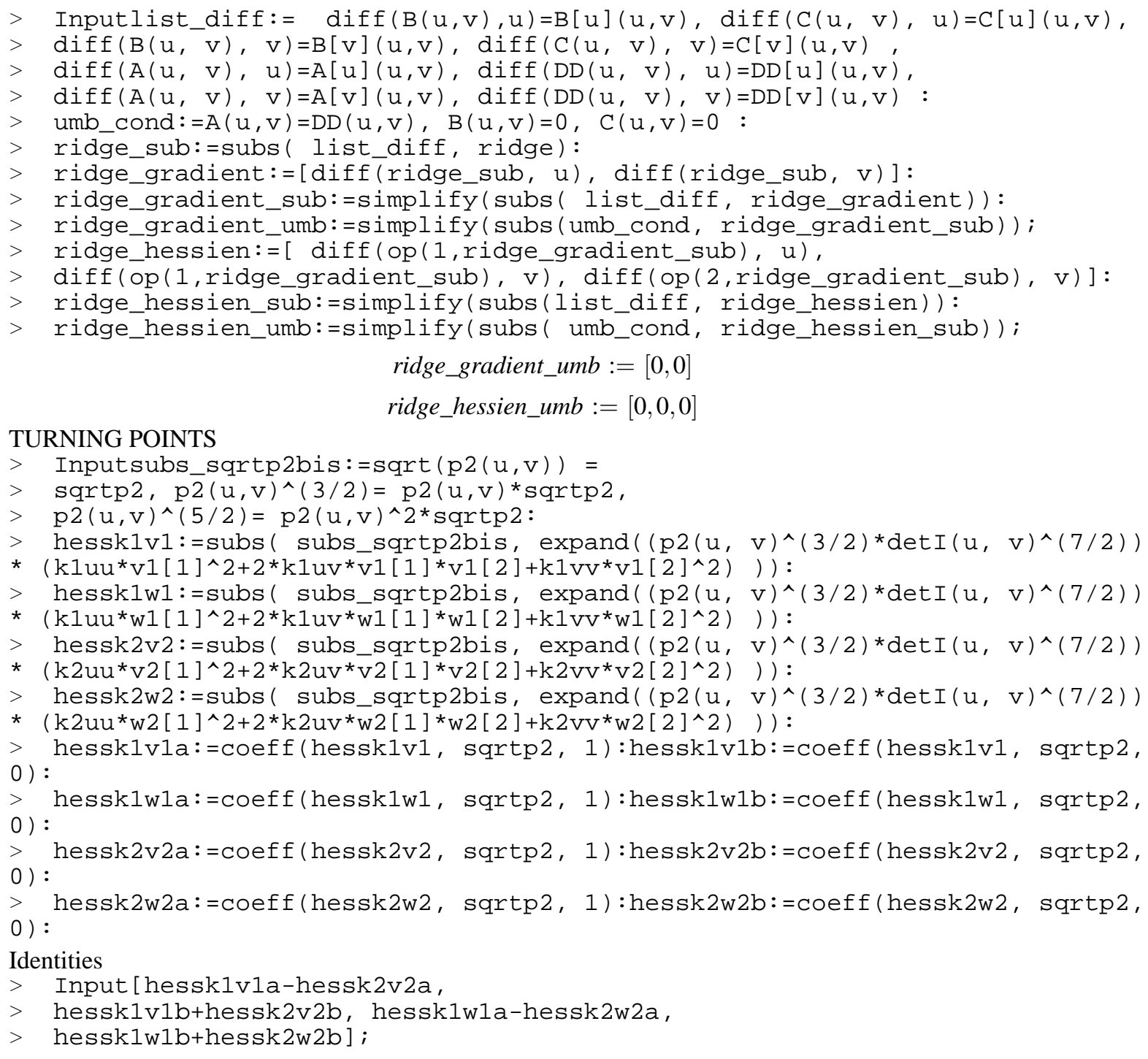

Definition of alpha, beta, alphabis, betabis: one has hessk1v1= alpha*sqrt $(\mathrm{p} 2(\mathrm{u}, \mathrm{v}))+$ beta; hessk2v2=alphabis*sqrt(p2(u,v)) -betabis

alpha, beta, alphabis, betabis are fct of A,B,C,DD, detI and first and second derivatives

$>$ alpha:=simplify ( $\operatorname{subs}($ subs_p2, hessk1vla )):

alphabis:=simplify ( subs( subs_p2, hessk1w1a)):

beta:=simplify ( subs ( subs_p2, hessk1v1b)) :

betabis:=simplify ( subs ( subs_p2, hessk1w1b)):

turn , turn_B and turn_C are fct of A,B,C,D,DetI and first and second derivatives

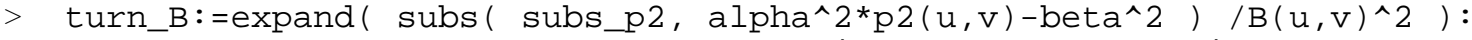

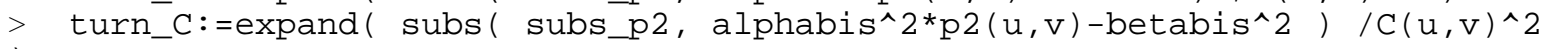

) :

$>$ turn_AD:= simplify $(2 *(a l p h a b i s * b e t a-a l p h a * b e t a b i s) /(-A(u, v)+D D(u, v))$

) :

Equivalence of equations

$>$ [turn_B-turn_C,turn_B-turn_AD] ;

$[0,0]$

Final equation, in each term of turn, there are 10 terms amongst A,B,C,D and 4 times DetI and 4 first derivatives ( $2 *$ first derivative $=2$ nd derivative $)$ 
$>$ turn:=simplify ( turn_AD/ content (turn_AD) ) : [whattype (turn), nops(turn), op (1, turn)] ;

$\left[{ }^{\prime}{ }^{`}, 17266,256 B(u, v)^{3} \operatorname{det} I(u, v)^{4} A(u, v) \frac{\partial}{\partial u} D D(u, v) \frac{\partial}{\partial u} B(u, v) C(u, v)^{2} \frac{\partial^{2}}{\partial u^{2}} A(u, v) D D(u, v)\right]$ 